\title{
Effective Larval Foraging in Large, Low-Diet Environments by Anopheles gambiae
}

\author{
A. C. Sutcliffe ${ }^{1,2}$ and M. Q. Benedict ${ }^{1,3}$ \\ ${ }^{1}$ Centers for Disease Control and Prevention (CDC), Atlanta, GA 30333, USA \\ ${ }^{2}$ Atlanta Research \& Education Foundation (AREF), 1670 Clairmont Road (151F), Decatur, GA 30033, USA \\ ${ }^{3}$ Dipartimento di Medicina Sperimentale e Scienze Biochimiche, Università Degli Studi di Perugia, Via del Giochetto, \\ 06122 Perugia, Italy \\ Correspondence should be addressed to M. Q. Benedict, mqbenedict@yahoo.com
}

Received 11 August 2011; Accepted 17 October 2011

Academic Editor: G. B. Dunphy

Copyright () 2012 A. C. Sutcliffe and M. Q. Benedict. This is an open access article distributed under the Creative Commons Attribution License, which permits unrestricted use, distribution, and reproduction in any medium, provided the original work is properly cited.

\begin{abstract}
Adult mosquito size is constrained by conditions experienced in the larval stage including the amount and quality of diet. The energy expended collecting diet depends partly on its concentration, the water depth, and the mosquito species. In order to better understand these interactions, individual Anopheles gambiae s.s. Giles were cultured to the adult stage in three types of experiments in which one of the following conditions was fixed and the other two were varied: water volume, diet amount, and diet concentration. In addition to survival, days of development to pupation and wing length were determined. The same outcomes were measured in experiments for which special containers were constructed that allowed the detection of chemical and tactile interactions. Larvae were able to develop to adulthood in volumes as great as $30 \mathrm{~mL} / \mathrm{larva}$ when diet was added at an average rate of only $7 \mu \mathrm{g} / \mathrm{mL} /$ day. The results demonstrate effective foraging in large low-diet volumes far above what had previously been estimated.
\end{abstract}

\section{Introduction}

Anopheline larvae develop in habitats that are often transient, poor in organic matter, and which vary greatly in size (e.g., $[1,2])$. Because the density of larvae also varies greatly, competition for the most limiting factor in habitats-the amount of food-has a strong effect on larval survival [36 ]. In addition to the limits placed on numerical habitat productivity by diet abundance and quality variation, the size of larval and adult mosquitoes is also irreversibly affected $[7,8]$. Size in turn affects vector life history: larger female mosquitoes are more fecund [7, 9], longer lived [10], and more likely to develop eggs from one blood meal [11]. Size is also related to the likelihood of developing infectious viruses $[12,13]$, though the effect on malaria parasite abundance is less clear $[14,15]$. Larger females are also more likely to be mated [16]. Moreover, the physical characteristics of how food is made available have measurable effects: water volume, surface area, and depth [8].
Intraspecific competition during the larval stages also influences adult mosquito body size, at least in part due to effects on diet abundance. It has been shown that increasing larval densities lead to longer development and reduced body size in certain Anopheles species [8, 17-19]. These interactions have been divided into physical and chemical interference [20]. How various kinds of interference individually influence larval development and adult size is poorly understood in spite of numerous studies, for example, [2123]. Chemical growth-retarding factors have been identified in Aedes and Culex spp. (discussed in [24]), though only one instance of these in an anopheline has been observed [18].

In order to better understand feeding and development dynamics of Anopheles gambiae, we used a simplified system to determine the effects of diet amount, concentration, and water volume on growth rate and size while considering survival as a secondary objective. By measuring growth outcomes with a reduced number of independent variables in the absence of intraspecific interaction, we determined 
TABLe 1: Daily rate of diet in three experiment types with "Day 1" being when L3s were transferred to dishes.

\begin{tabular}{|c|c|c|c|c|c|c|c|c|c|c|c|c|c|c|}
\hline \multirow[t]{2}{*}{ Day } & \multicolumn{5}{|c|}{$\begin{array}{c}\text { Fixed water volume }(\mu \mathrm{g} / \text { larva in } 30 \mathrm{~mL}) \\
\text { Relative amount of diet }\end{array}$} & \multicolumn{5}{|c|}{$\begin{array}{c}\text { Fixed diet concentration }(\mu \mathrm{g} / \text { larva }) \\
\text { Water volume }(\mathrm{mL})\end{array}$} & \multicolumn{4}{|c|}{$\begin{array}{l}\text { Fixed diet amount }(\mu \mathrm{g} / \mathrm{mL}) \\
\text { Water volume }(\mathrm{mL})\end{array}$} \\
\hline & 0.1 & 0.5 & 1 & 2 & 3 & 0.2 & 1.0 & 5.0 & 11.0 & 30 & 1.0 & 5.0 & 11.0 & 30.0 \\
\hline 1 & 60 & 300 & 600 & 1200 & 1800 & 8 & 40 & 200 & 440 & 1200 & 440 & 88 & 40 & 15 \\
\hline 2 & 80 & 400 & 800 & 1600 & 2400 & 11 & 53 & 267 & 587 & 1600 & 580 & 116 & 53 & 19 \\
\hline 3 & 100 & 500 & 1000 & 2000 & 3000 & 13 & 67 & 333 & 733 & 2000 & 740 & 148 & 67 & 25 \\
\hline 4 & 120 & 600 & 1200 & 2400 & 3600 & 16 & 80 & 400 & 880 & 2400 & 880 & 176 & 80 & 29 \\
\hline 5 & 140 & 700 & 1400 & 2800 & 4200 & 19 & 93 & 467 & 1027 & 2800 & 1020 & 204 & 93 & 34 \\
\hline 6 & 160 & 800 & 1600 & 3200 & 4800 & 21 & 107 & 533 & 1173 & 3200 & 1180 & 236 & 107 & 39 \\
\hline 7 & 180 & 900 & 1800 & 3600 & 5400 & 24 & 120 & 600 & 1320 & 3600 & 1320 & 264 & 120 & 44 \\
\hline 8 & 200 & 1000 & 2000 & 4000 & 6000 & 27 & 133 & 667 & 1467 & 4000 & 1460 & 292 & 133 & 49 \\
\hline 9 & 220 & 1100 & 2200 & 4400 & 6600 & 29 & 147 & 733 & 1613 & 4400 & 1620 & 324 & 147 & 54 \\
\hline
\end{tabular}

the potential responses of individuals to conditions ranging from low to extremely high diet amounts and concentrations. We also devised and tested an experimental system to distinguish the effects of chemical and physical larval interactions.

\section{Materials and Methods}

The mosquito stock used for this study was obtained from the Malaria Research and Reference Reagent Resource Center (MR4) vector activity at the CDC in Atlanta, GA, USA: Anopheles gambiae G3 (MRA-112). All stages were reared at $27 \pm 2^{\circ} \mathrm{C}$ using typical methods [25] on a diet of Koi Floating Blend (Aquaricare, Victor, NY. product no longer available). Except for the baker's yeast fed to hatching larvae, this diet was used in all experiments.

Three types of larval culture experiments were conducted by fixing one of the three factors of interest in each type of experiment successively: (1) water volume, (2) diet concentration/mL, and (3) total diet amount/larva. The order of experiments presented here reflects the order in which the tests were conducted. Outcomes from a given experiment determined the fixed parameter implemented in the subsequent experiment. Because we manipulated the diet concentration and amount by changing the volume of water, we will describe the outcomes as a function of water volume in those experiments.

On the first day of each trial, approximately 900 eggs were placed in $300 \mathrm{~mL}$ of water containing $3 \mathrm{~mL}$ of $2 \% \mathrm{w} / \mathrm{v}$ baker's yeast in a $34 \times 25 \mathrm{~cm}$ plastic tray. On day three, 300 larvae were counted into a new pan containing $300 \mathrm{~mL}$ of $0.3 \% \mathrm{w} / \mathrm{v}$ artificial sea salt (Instant Ocean, Aquarium Systems, Mentor, OH, USA) in reverse-osmosis/deionized (RODI) purified water and $3 \mathrm{~mL}$ of $0.2 \% \mathrm{w} / \mathrm{v}$ suspension of finely ground Koi diet in RODI water. Trays were monitored and fed with $3 \mathrm{~mL}$ of $0.2 \% \mathrm{w} / \mathrm{v}$ finely ground diet daily. On the first day that the third stage larvae (L3s) appeared, they were transferred to polystyrene dishes according to details of each experiment (see Section 3). Water in all dishes was $5 \mathrm{~mm}$ deep, and the dishes were covered with the supplied transparent lid to reduce evaporation. Subsequently, larvae were fed with various volumes of $0.2 \%$ w/v finely ground diet in water (Table 1), the concentration of which was maintained uniform by vigorous manual shaking between each pipetting. Dishes were monitored every morning at which time the dish, water, and diet were changed to eliminate waste and diet accumulation. Each experiment was performed three times, and in each, 10 individuals were usually observed. Pupae were transferred to an emergence container containing $30 \mathrm{~mL}$ of RODI water. Approximately, two days after emergence, adults were killed by freezing, sex was determined, and one wing was removed and photographed after which length was measured using size-calibrated ImageJ software [26]. Length was measured from the base of the radial vein to the most distant portion of the wing tip, excluding setae. The larval duration and wing length were analyzed using a general linear model with Minitab (State College, PA, USA).

For the interaction experiments, three dish types were used and, for reasons that will be evident, were named "undivided," "porous," and "divided." All were modifications of standard $90 \mathrm{~mm}$ diameter polystyrene Petri dishes (the bottom of which is actually $88 \mathrm{~mm}$ diameter) described above which for these experiments were called "undivided." The porous dish was constructed by gluing strips of $2 \mathrm{~mm}$ thick hydrophilic, porous polyethylene (Small Parts Inc., Miami, FL, USA) with a pore size of $90-130 \mu$ to the bottom and sides of the undivided dish to create four equal sized compartments. The pore size is sufficiently large that water can pass through the plastic sheet. The glue used for all construction was PVC "hot glue." The divided dish was of the same overall dimensions and material but manufactured to have four equal sized compartments divided by solid partitions. Both the divided and undivided dishes had a piece of the same porous polyethylene hotglued to the bottom of the dish to preserve a uniform volume and materials content with the porous dish. In the divided and porous dishes, one larva was added to each compartment and four larvae were added to the undivided dish. Water was not changed throughout the trial, and food was added every other morning in equal volumes to each chamber of the divided dishes or 4 -fold as much to the undivided dish. Water volume $(30 \mathrm{~mL} / \mathrm{dish})$, food amount 


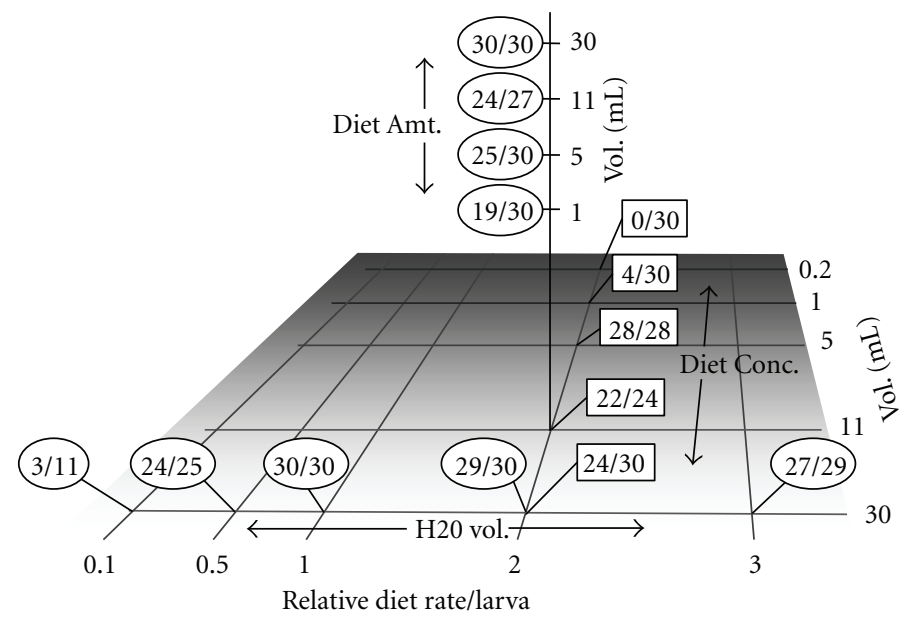

FIgURE 1: Graphical description of the experimental conditions and survival data. The three fixed conditions (water volume, diet concentration, and diet amount) are indicated on the axis corresponding to the experimental values. The $Z$ axis presents the volumes of water used and crosses the $X Y$ plane at the diet amount used. Values in the boxes and ovals are the number of larvae that successfully pupated divided by the total number in the experiment.

TABLE 2: Analysis by GLM of three experiments with a single-fixed variable (type III values).

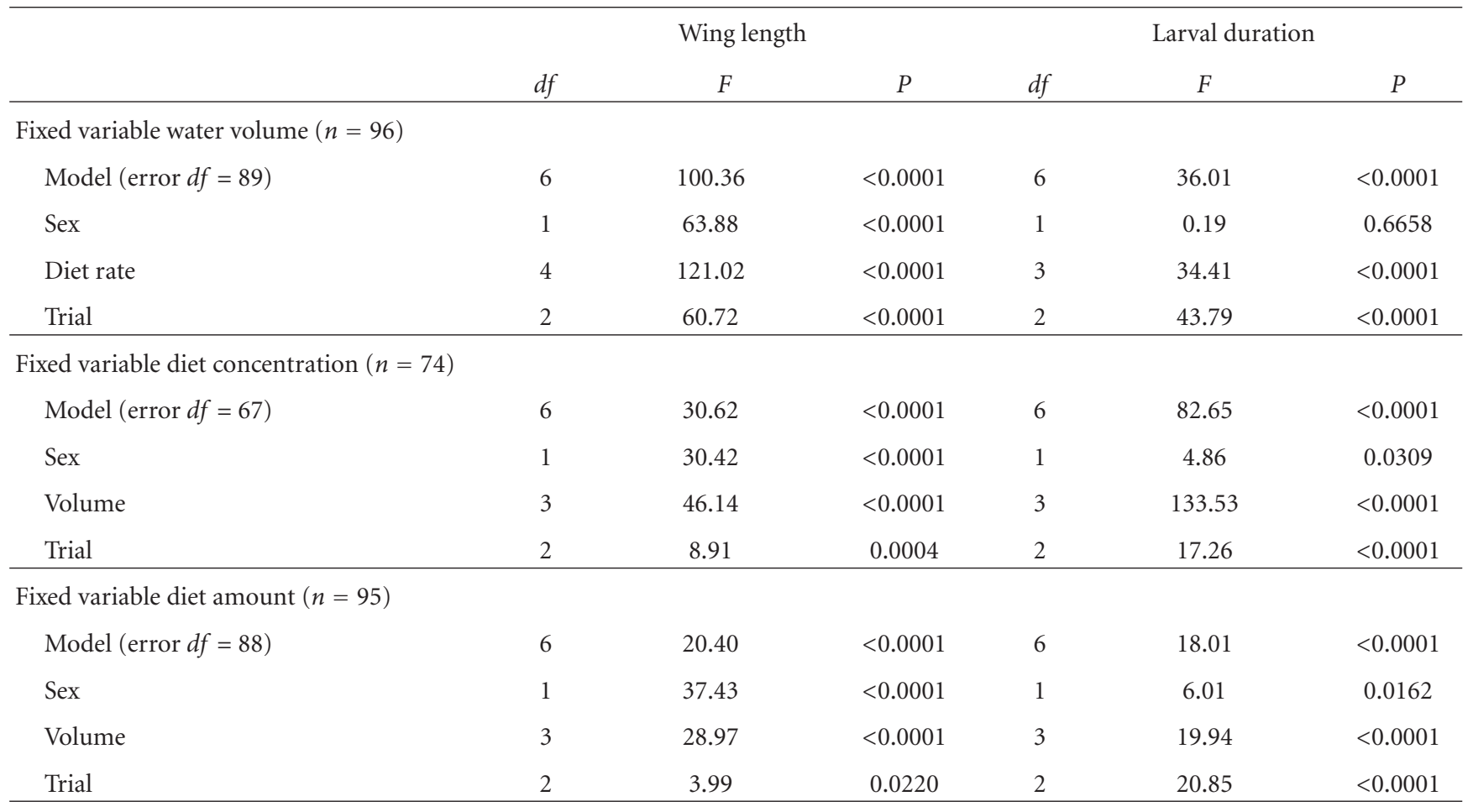

(1600 $\mu \mathrm{g} /$ larva/alternating day) and number of larvae per dish remained constant throughout the experiment. Only dishes in which all four larvae pupated were included in the analysis. Three trials were conducted with 20 individuals (five dishes) in each treatment group. Data was analyzed using SAS software (Cary, NC, USA) and Minitab using an alpha of 0.05. Pairwise comparisons of general linear model (GLM) outcomes were performed using Tukey's method.

\section{Results}

The conditions that were chosen for the experiments and their relationship to one another is shown in Figure 1. With few exceptions, sex and the independent variable had highly significant effects on both wing length and larval duration (Table 2). Therefore, we analyzed and graphed sex separately regardless of whether both were significant in the overall 


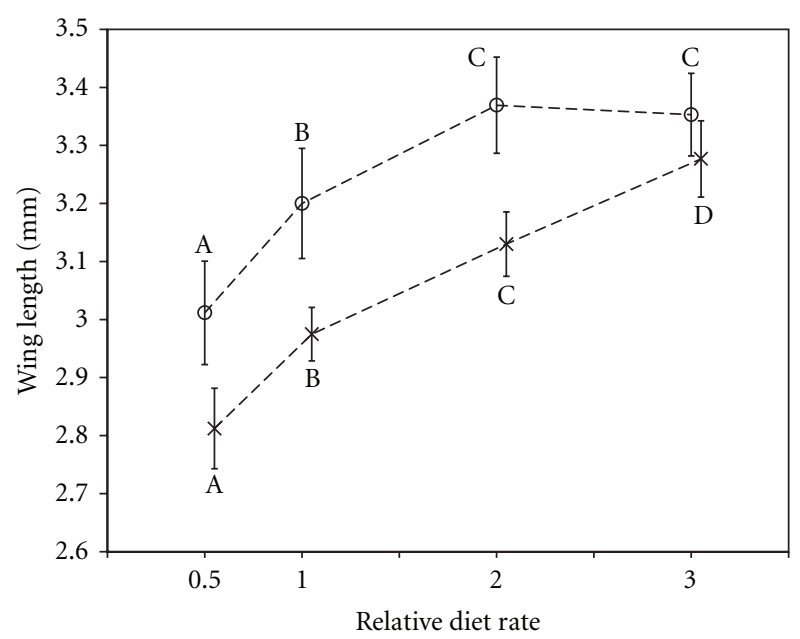

○ Female

$\times$ Male

FIgURe 2: Mean mosquito wing lengths in a constant water volume of $30 \mathrm{~mL}$. Larvae were provided diet in multiples of the standard diet rate. Dotted lines are for clarity of data groupings and are not intended to represent intermediate values in this or in other figures. Letters above and below data indicate significantly different values for females and males when analyzed by sex. Error bars are $95 \%$ CI of the mean. In this and all other graphs, male data are shifted rightward for clarity.

model. All models were highly significant. Trial was significant; however, the culture condition trends are so strong that this does not obscure the conclusions.

Survival in most experiments was high (Figure 1). Of 384 individual larvae used in these experiments, 76\% (289) pupated. Those that did not were clustered in specific experimental conditions which we will expand upon in context below.

3.1. Constant Water Volume. In the first set of experiments, diet concentration was varied (concomitantly with diet amount), while the dish type (standard $90 \mathrm{~mm}$ polystyrene Petri dishes) contained a constant water volume of $30 \mathrm{~mL}$. The relative amount of diet was increased daily according to a scale suggested previously [25]. Because the amount of diet fed daily accelerated in all experiments (Table 1), we will refer to the amount of diet as rates relative to those described in that table, that is, 0.1-0.5-, 1-, 2-, and 3-fold.

Of 125 larvae placed individually in dishes, 12 did not survive to pupation, eight of which were in the lowest (0.1fold) diet-level dishes. Of the 11 larvae tested under the 0.1 -fold conditions, only two females developed, so this condition was removed from analysis of duration and wing length. Until the day of death, larvae in this group survived from $11-15$ days.

Male wing length increased significantly as the diet increased from 2 - to 3 -fold, whereas maximum female wing length was reached at 2-fold (Figure 2). Maximum wing length was reached at lower diet concentrations for females than for males indicating that they are able to forage and/or

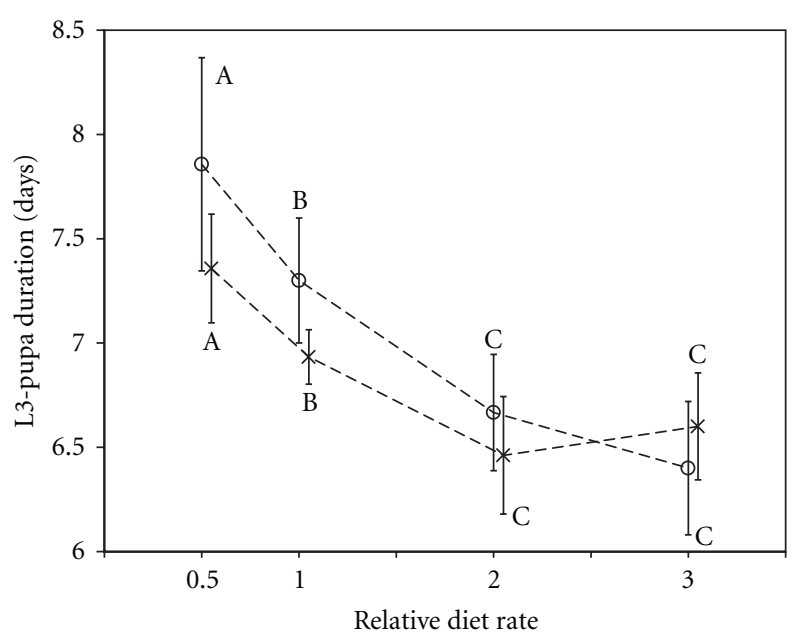

$$
\begin{aligned}
& \bigcirc \text { Female } \\
& \times \text { Male }
\end{aligned}
$$

Figure 3: Mean larval durations in a constant water volume of $30 \mathrm{~mL}$. Larvae were provided diet in multiples of the standard diet rate. Letters above and below indicate significantly different values for females and males when analyzed by sex. Error bars are 95\% CI of the mean.

assimilate diet more efficiently. In contrast, minimal larval duration was achieved for both sexes at the 2-fold level (Figure 3). Based on these experiments, the 2-fold diet level was considered nonrestrictive and was chosen for the next stage of experiments.

3.2. Constant Diet Concentration. In these experiments, the diet concentration $/ \mathrm{mL}$ water was held constant while the water volume was diminished. Volumes were manipulated to maintain the constant depth of $5 \mathrm{~mm}$ by using dishes of various inside diameters: $3,16,35,53 \mathrm{~mm}$ in addition to the $88 \mathrm{~mm}$ dish used previously. The water volumes were $0.2,1.0$, 5.0, 11.0, and $30.0 \mathrm{~mL}$. The diet rates are listed in Table 1 . Because "volume" is more easily visualized, we will refer to the outcomes according to this variable.

Pupae formed in all volumes except $0.2 \mathrm{~mL}(n=30)$. In this volume, many lived longer than 20 days though the average was 12.2 days. This demonstrates that diet was not adequate to allow pupation or that the physical size restriction in some way prevented normal development. Of the 30 larvae cultured in $1 \mathrm{~mL}\left(2 \mathrm{~cm}^{2}\right.$ surface area), only four pupated ( 2 males, 2 females), and those that did not died at 11.7 days on average, but none lived longer than 15 days. Due to the small number, the four survivors were eliminated from further analysis. The individuals that died in the specific cases described in the first two experiments above account for 65 of 95 of the total that failed to pupate in all experiments.

In the remaining treatments, sex, volume, and trial affected wing length and larval duration (Table 2, Figures 4 and 5). Increases in wing length and development rates were observed with volume increases from 5.0 to $11.0 \mathrm{~mL}$. While the differences in these outcomes between the 11.0 and $30.0 \mathrm{~mL}$ volume were not significant, the trends suggest 


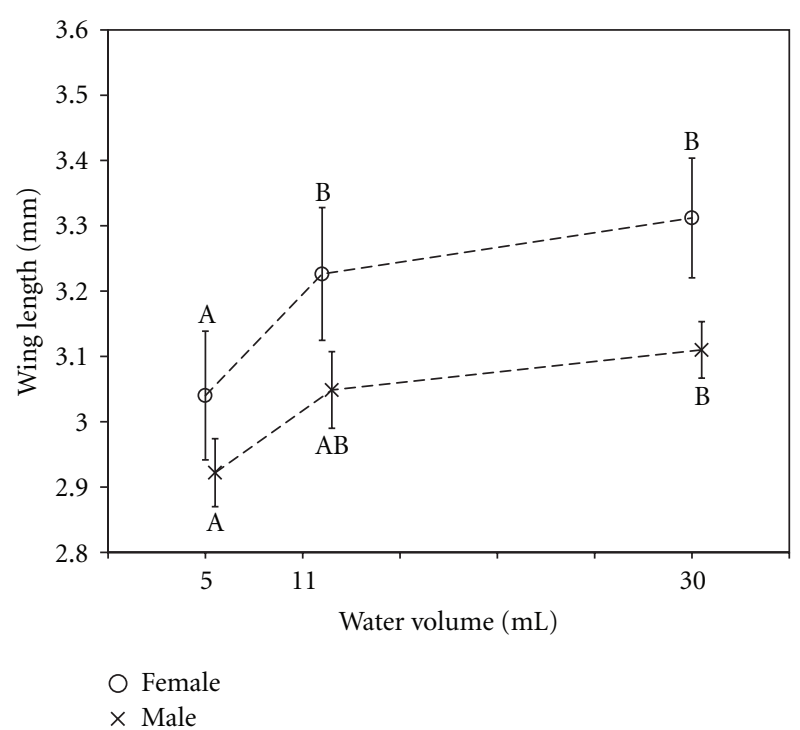

Figure 4: Mean mosquito wing lengths when provided the 2fold diet schedule (Table 1) but in reduced amounts to maintain a constant diet concentration per $\mathrm{mL}$ in the various dishes. Letters above and below indicate significantly different values when analyzed by sex. Error bars are $95 \%$ CI of the mean.

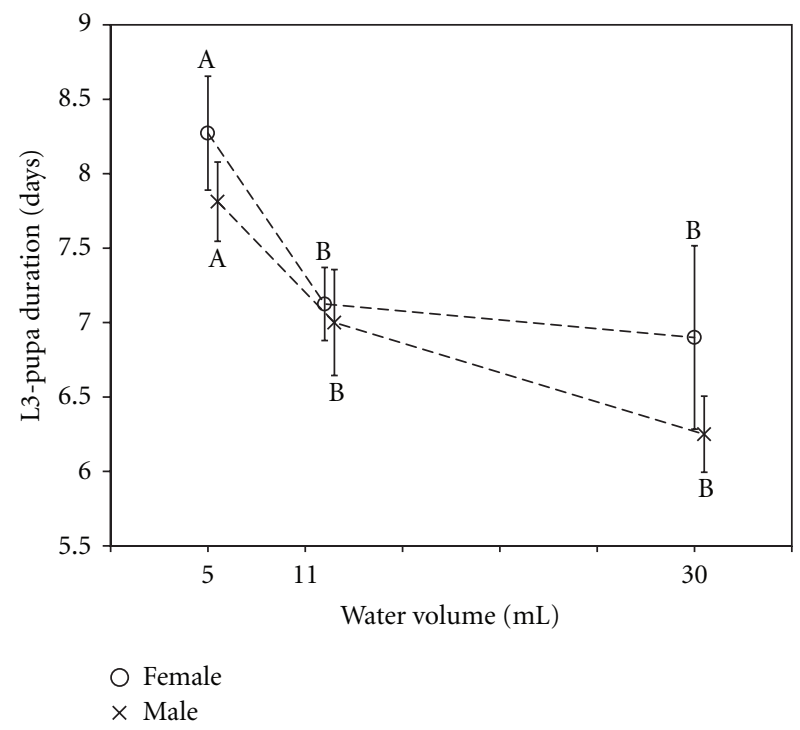

FIGURE 5: Mean larval durations when provided the 2-fold diet schedule (Table 1) but in reduced amounts to maintain a constant diet concentration per $\mathrm{mL}$ in the various dishes. Letters above and below indicate significantly different values when analyzed by sex. Error bars are $95 \% \mathrm{CI}$ of the mean.

that a small increase may be correlated with larger volume that was not detected in our experiments. Based on these experiments, the amount of diet in the $11.0 \mathrm{~mL}$ treatment was chosen for the next series.

3.3. Constant Diet Amount. The diet weight fed in the $11.0 \mathrm{~mL}$ treatment was provided in higher and lower concentrations by using the various dish sizes described above,

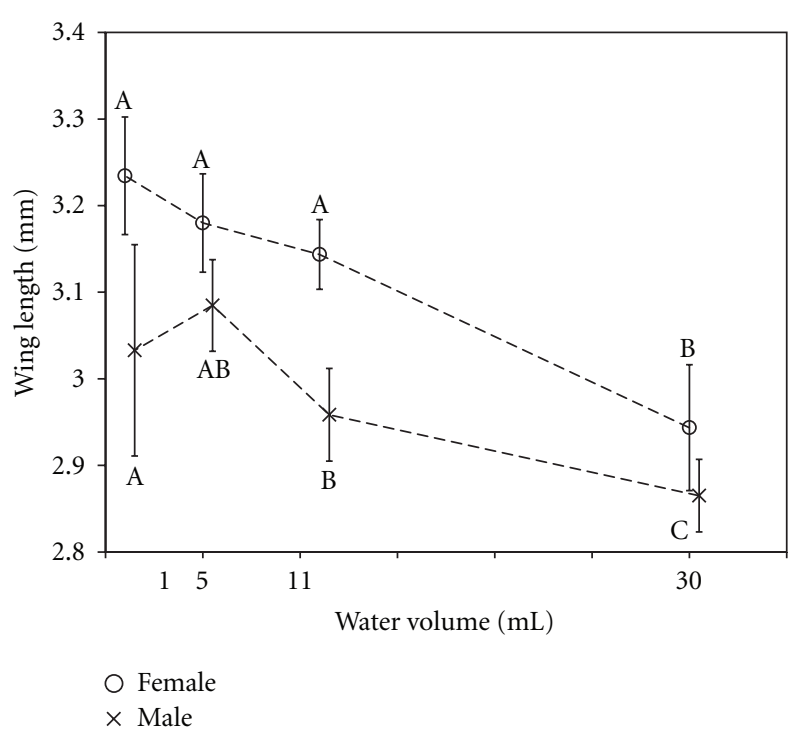

FIGURE 6: Mean mosquito wing lengths by sex when given a constant diet weight per dish. Water volume and diet concentration varied. Letters above and below indicate significantly different values when analyzed by sex. Error bars are $95 \%$ CI of the mean.

with the exception that the $0.2 \mathrm{~mL}$ dish was not included because no larvae had survived previously in that volume. In these experiments, a trend of mortality as a function of diet concentration existed. There were 11 deaths in the $1.0 \mathrm{~mL}$ dishes, five in the $5.0 \mathrm{~mL}, 3$ in the 11 , and 0 in the $30 \mathrm{~mL}$. This association (ANOVA $F=78.64,3 d f, P=$ 0.012 ) and strong correlation of mortality with increased diet concentration and diminished water volume $(S=0.8961$, $R^{2}=96.3 \%$ ) indicates a negative effect on survival of a small volume and/or a toxic effect of the diet itself. This negative trend on mortality was not reflected in reduced wing length and increased larval duration of the survivors (Figures 6 and 7 , resp.), characters for which positive effects of diminishing the volume, and increasing the concentration of diet were observed.

3.4. Tactile and Chemical Interactions. In order to detect interaction effects of larvae, we cultured individuals in containers which would allow or preclude either chemical and/or tactile effects. Both sexes were pooled for analysis by GLM initially. Dish type and sex both had significant effects on wing length $(F=3.77,2 d f, P=0.025$ and $F=8.25$, $1 d f, P=0.005$, resp.) but not larval duration. When females and males were pooled, the average wing length of adults from larvae cultured in the divided dish was significantly shorter than of those cultured in either the porous or open dish (Figure 8). When wing length and larval duration were analyzed by sex, no significant differences in wing length or duration as a function of dish type were observed.

\section{Discussion}

Numerous methods have been used to determine the feeding rates of larvae: suspended particle removal [27], gut bolus 


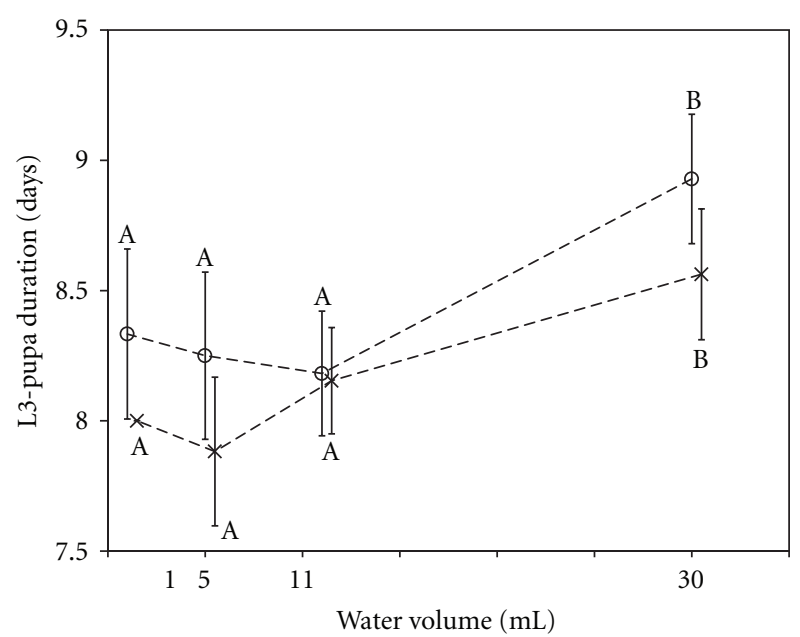

O Female

$\times$ Male

FIGURE 7: Mean larval duration when given a constant diet weight of diet per dish. Water volume and diet concentration varied. Letters above and below indicate significantly different values when analyzed by sex. All males $(n=7)$ cultured in $1.0 \mathrm{~mL}$ pupated on the eighth day. Error bars are $95 \%$ CI of the mean.

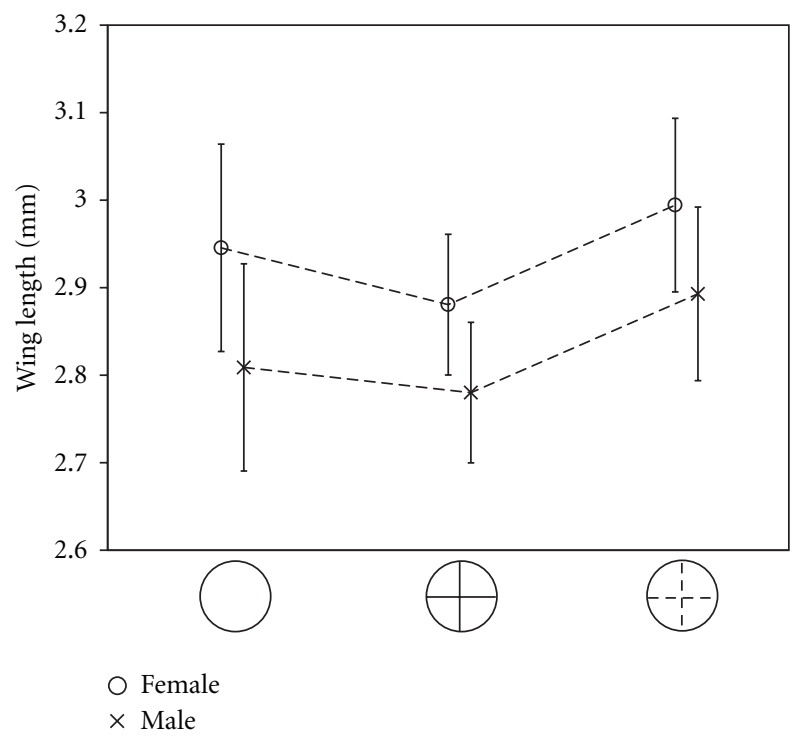

Figure 8: Mean mosquito wing lengths for three different dish types with the same water volume and food amount per larva. Symbols for the three dish types are shown from left to right: open, solid divided, and porous divided. Error bars are 95\% CI of the mean.

displacement [28] and mouthpart activity [29]. The methods of presenting the diet (and surrogate indicators such as charcoal and latex beads) have included in solution [30], on the surface, and in slurries [8]. All are appropriate presentations for anophelines which feed on the surface bacterioneuston, drink, graze on the bottom and scrape particles (discussed extensively in [24]). We do not know how much of the diet we provided was deposited in and collected from these different locations, though sedimented particles were visually most apparent. All dishes contained water of the same shallow depth, and no change occurred as water volume and food concentration were varied. Therefore, the diet could be easily obtained by the larvae which are often observed feeding on the surface in the stereotypical anopheline fashion, but which also readily reached the bottom. Changing the diet daily reduced the nutritional contribution of microorganisms, prevented diet accumulation, and reduced the effects of diet degradation.

In all experiments, there was a strong positive correlation between development rate and wing length, but we did not explore this in detail. This relationship has been reported previously (e.g., $[19,31,32])$. Briefly, faster development is correlated with increased wing length.

The first three experiments reported here are unlike many (e.g., $[3,8,23])$ in which larvae were allowed to interact and in which diet and water were not replaced on a daily basis. By adjusting the volumes and amounts of diet, we detected growth-limiting effects of extremely low diet concentrations, that is, the concentration of diet at which expenditure of energy required for homeostasis and foraging for food outweighed its nutritional benefits. When cultured in $30 \mathrm{~mL}$, survival was extremely low unless larvae were provided at least $7 \mu \mathrm{g} / \mathrm{mL}$ of diet per day (equal to $220 \mu \mathrm{g} /$ larva/day based on the amount provided on day 9 and thereafter). It is possible that the low amount of diet provided in the earlier development stages $(2 \mu \mathrm{g} / \mathrm{mL} /$ day $)$ precluded later survival. Nonetheless, it is remarkable that any development occurred at all when diet was provided in such low concentrations and demonstrates that larvae can assimilate diet effectively even when it is very dilute. Because all motion expends energy, foraging activity does not come without cost in size and growth rate. This is demonstrated by significant increases in size that were observed up to diet concentrations near $150 \mu \mathrm{g} / \mathrm{mL}$ above which point little benefit of increasing the concentration was observed.

We chose to begin the experiments with the early third instar larvae. In our experience, survival under a wide range of conditions after the L3 stage is high-in contrast to relatively delicate L1s-thus increasing the probability of obtaining useful larval development duration and wing length data. Most of the mass of larval mosquitoes accumulates during the L3 and L4 stages. This choice may have influenced the outcomes we observed, and that of the minimum diet required for development is probably the most sensitive. The minimum amount of diet necessary for survival that we estimated here $(220 \mu \mathrm{g})$ reflects only what was assimilated after the beginning of the third stage. However, in separate estimates using two different methods with a sibling species of An. gambiae, An. arabiensis, estimates of the minimum amount of the same diet required for development from hatching were 202 and $263 \mu \mathrm{g}$ per larva [19].

Considering the amount of diet required for survival in this study, it is possible to obtain indirect estimates of the volume of water that larvae can filter. Aly [27] estimated anopheline filtration rates of $33-55 \mu \mathrm{L} / \mathrm{larva} / \mathrm{h}$ for An. albimanus and An. quadrimaculatus, respectively. If the rate on the high end of this estimate were applied to our 
experimental system and species, larvae could process only $1.3 \mathrm{~mL}$ of water per day, meaning that only approximately, $10 \mu \mathrm{g}$ of diet/day could be consumed in our low concentration experiments-an unrealistically small amount that is not consistent with survival. Our results demonstrate that the method used to determine filtration volume estimates must have resulted in gross underestimates, possibly due to a low efficiency of latex bead collection per volume of water actually processed. We suggest that the volume of water that larvae can process is not a very realistic indicator of feeding efficiency: larvae certainly reduce overall effort by focusing on high-value particles that are encountered rather than randomly processing water.

The second set of experiments, in which the diet concentration was fixed, determined the amount of diet that is necessary when provided at a saturating concentration (determined in the fixed volume experiments). Consistent with the first experiments, we observed almost no survival below $150 \mu \mathrm{g} /$ larva/day (based on day 9 values) even when larvae were provided this amount in one $\mathrm{mL}$ which would require little foraging. The similarity between the minimum amount of diet necessary for survival in the first experiments $(220 \mu \mathrm{g} /$ larva in $30 \mathrm{~mL})$ and those in the second set $(150 \mu \mathrm{g} /$ larva in $1 \mathrm{~mL})$ demonstrates that foraging is extremely efficient with regard to survival to pupation. The difference between these values provides an estimate of the nutrition required for foraging in $30 \mathrm{~mL}$, approximately $70 \mu \mathrm{g}$ of diet per day per larva.

On the other end of the spectrum of conditions tested in these experiments, maximal wing length and most rapid development occurred when larvae were each provided $11.0 \mathrm{~mL}$ of water containing $1600 \mu \mathrm{g}$ of diet-conditions that are approximately 10 -fold more spacious and diet-rich than the minimum required for survival. Volumes and diet levels greater than this provided no measurable benefit on growth.

The fixed diet amount experiments returned to the question of foraging efficiency developed in the fixed water volume experiments. In these, diet was provided in the $1 \mathrm{~mL}$ volume at a concentration more than seven times as high as the highest feeding rate provided in the first (constant volume) experiments. A negative effect of increased diet concentration on survival was observed suggesting either a toxic effect of the diet, harmful effects of waste concentration, or an inability to utilize extremely high concentrations.

It has been established that culicine chemical factors are produced during the larval stage that can inhibit larval development (see [24]), and there is some support for this in An. stephensi [18]. The undivided and the porous dish both allowed chemical interaction, while the undivided dish also allowed tactile interaction. Under these conditions, chemical interaction resulted in larger size. When neither interaction was possible (divided dishes), adults were smaller. We can imagine only one reason: sensing the presence of potential competitors may stimulate more active foraging. Such experiments are admittedly open to criticism for several reasons: some differences existed between the dish volumes or materials, the diet concentration was inappropriate, and so forth. Every effort was made to match the materials and volumes though the geometry could admittedly have an effect. The experiments do provide provocative preliminary results and a method for developing systems for testing such phenomena further.

While natural production of healthy mosquitoes is undesirable for public health, artificial production is beneficial for laboratory experiments and repositories such as the Malaria Research and Reference Reagent Resource Center (MR4, http://www.mr4.org/), genetic control efforts such as the sterile insect technique [33], and for meaningful comparisons between wild and laboratory-reared specimens, for example, $[34,35]$. The information we have developed here will provide guidance for optimal conditions for such activities, though, to the best of our knowledge, typical densities in production facilities will be much higher than the optimal ones we observed.

Consistent with their "clean water" reputation, these experiments reveal that An. gambiae larval foraging activity is extremely efficient and spatially extensive, and that they are able to utilize even very low concentrations of diet. The extended development times that we observed when diet was limiting also mean that surveys of larval habitats may overestimate the standing population as many such sites will contain very long-lived larvae, few of which survive to adulthood, a suspicion observed in some field studies [36].

\section{Acknowledgments}

The authors appreciate the support of the Malaria Research and Reference Reagent Resource Center (MR4) for the An. gambiae stock. They also appreciate the helpful advice and statistical analysis performed by Jacqueline Roberts of the CDC.

\section{References}

[1] T. B. Ageep, J. Cox, M. M. Hassan et al., "Spatial and temporal distribution of the malaria mosquito Anopheles arabiensis in northern Sudan: influence of environmental factors and implications for vector control," Malaria Journal, vol. 8, no. 1, article 123, 2009.

[2] J. E. Gimmg, M. Ombok, L. Kamau, and W. A. Hawley, "Characteristics of larval anopheline (Diptera: Culicidae) habitats in western Kenya," Journal of Medical Entomology, vol. 38, no. 2, pp. 282-288, 2001.

[3] W. K. Reisen, "Intraspecific competition in Anopheles stephensi Liston," Mosquito News, vol. 35, no. 4, pp. 473-482, 1975.

[4] T. S. J. Bellows, "The descriptive properties of some models for density dependence," Journal of Animal Ecology, vol. 50, no. 1, pp. 139-156, 1981.

[5] H. Briegel, "Physiological bases of mosquito ecology," Journal of Vector Ecology, vol. 28, no. 1, pp. 1-11, 2003.

[6] S. A. Juliano, "Population dynamics," Journal of the American Mosquito Control Association, vol. 23, no. 2, supplement, pp. 265-275, 2007.

[7] H. Briegel, "Fecundity, metabolism, and body size in Anopheles (Diptera: Culicidae), vectors of malaria," Journal of Medical Entomology, vol. 27, no. 5, pp. 839-850, 1990.

[8] S. E. Timmermann and H. Briegel, "Water depth and larval density affect development and accumulation of reserves in laboratory populations of mosquitoes," Bulletin of the Society of Vector Ecology, vol. 18, no. 2, pp. 174-187, 1993. 
[9] E. O. Lyimo and W. Takken, "Effects of adult body size on fecundity and the pregravid rate of Anopheles gambiae females in Tanzania," Medical \& Veterinary Entomology, vol. 7, no. 4, pp. 328-332, 1993.

[10] W. Takken, M. J. Klowden, and G. M. Chambers, "Effect of body size on host seeking and blood meal utilization in $A n$ opheles gambiae sensu stricto (Diptera: Culicidae): the disadvantage of being small," Journal of Medical Entomology, vol. 35, no. 5, pp. 639-645, 1998.

[11] L. P. Lounibos, D. Couto Lima, R. Lourenço-de-Oliveira, R. L. Escher, and N. Nishimura, "Egg maturation in neotropical malaria vectors: one blood meal is usually enough," Journal of Vector Ecology, vol. 23, no. 2, pp. 195-201, 1998.

[12] B. W. Alto, M. H. Reiskind, and L. P. Lounibos, "Size alters susceptibility of vectors to dengue virus infection and dissemination," American Journal of Tropical Medicine and Hygiene, vol. 79, no. 5, pp. 688-695, 2008.

[13] S. N. Bevins, "Invasive mosquitoes, larval competition, and indirect effects on the vector competence of native mosquito species (Diptera: Culicidae)," Biological Invasions, vol. 10, no. 7, pp. 1109-1117, 2008.

[14] H. Hurd, J. C. Hogg, and M. Renshaw, "Interactions between bloodfeeding, fecundity and infection in mosquitoes," Parasitology Today, vol. 11, no. 11, pp. 411-416, 1995.

[15] J. M. Mwangangi, C. M. Mbogo, J. G. Nzovu et al., "Relationships between body size of Anopheles mosquitoes and Parasitology falciparum sporozoite rates along the Kenya coast," Journal of the American Mosquito Control Association, vol. 20, no. 4, pp. 390-394, 2004.

[16] F. M. Okanda, A. Dao, B. N. Njiru et al., "Behavioural determinants of gene flow in malaria vector populations: Anopheles gambiae males select large females as mates," Malaria Journal, vol. 1, no. 1, pp. 1-10, 2002.

[17] R. W. Merritt, R. H. Dadd, and E. D. Walker, "Feeding behavior, natural food, and nutritional relationships of larval mosquitoes," Annual Review of Entomology, vol. 37, no. 1, pp. 349-376, 1992.

[18] W. K. Reisen and R. W. Emory, "Intraspecific competition in Anopheles stephensi (Diptera Culicidae) II. The effects of more crowded densities and the addition of antibiotics," Canadian Entomologist, vol. 109, pp. 1475-1480, 1977.

[19] J. R. Gilles, R. S. Lees, S. M. Soliban, and M. Q. Benedict, "Density-dependent effects in experimental larval populations of Anopheles arabiensis (Diptera: Culicidae) can be negative, neutral, or overcompensatory depending on density and diet levels," Journal of Medical Entomology, vol. 48, no. 2, pp. 296 304, 2011.

[20] S. A. Juliano, "Species interactions among larval mosquitoes: context dependence across habitat gradients," Annual Review of Entomology, vol. 54, pp. 37-56, 2009.

[21] C. Dye, "Competition amongst larval Aedes aegypti: the role of interference," Ecological Entomology, vol. 9, no. 3, pp. 355-357, 1984.

[22] W. K. Reisen and R. W. Emory, "The effects of larval intraspecific competition on imaginal densities in Anopheles stephensi (Diptera: Culicidae): a laboratory evaluation," Canadian Entomologist, vol. 109, pp. 1481-1484, 1977.

[23] A. Mori, "Effects of larval density and nutrition on some attributes of immature and adult Aedes albopictus," Tropical Medicine, vol. 21, no. 2, pp. 85-103, 1979.

[24] A. N. Clements, The Biology of Mosquitoes: Development, Nutrition, and Reproduction, Chapman \& Hall, London, UK, 1992.
[25] M. Q. Benedict, "Care and maintenance of anopheline mosquito colonies," in The Molecular Biology of Insect Disease Vectors, J. M. Crampton, C. B. Beard, and C. Louis, Eds., pp. 2-12, Chapman \& Hall, New York, NY, USA, 1997.

[26] M. D. Abràmoff, P. J. Magalhães, and S. J. Ram, "Image processing with imageJ," Biophotonics International, vol. 11, no. 7, pp. 36-41, 2004.

[27] C. Aly, "Filtration rates of mosquito larvae in suspensions of latex microspheres and yeast cells," Entomologia Experimentalis et Applicata, vol. 46, no. 1, pp. 55-61, 1988.

[28] S. S. Rashed and M. S. Mulla, "Factors influencing ingestion of particulate materials by mosquito larvae (Diptera: Culicidae)," Journal of Medical Entomology, vol. 26, no. 3, pp. 210-216, 1989.

[29] R. H. Dadd, "Relationship between filtering activity and ingestion of solids by larvae of the mosquito Culex pipiens: a method for assessing phagostimulant factors," Journal of Medical Entomology, vol. 7, no. 6, pp. 708-712, 1970.

[30] R. H. Dadd, J. E. Kleinjan, and V. P. Sneller, "Development of several species of mosquito larvae in fully defined dietary media: preliminary evaluation," Mosquito News, vol. 37, no. 4, pp. 699-703, 1977.

[31] S. R. Carpenter, "Experimental test of the pupation window model for development of detritivorous insects," Ecological Modelling, vol. 23, no. 3, pp. 257-264, 1984.

[32] M. E. Gilpin and G. A. McClelland, "Systems analysis of the yellow fever mosquito Aedes aegypti," Fortschritte der Zoologie, vol. 25, no. 2-3, pp. 355-388, 1979.

[33] A. S. Robinson, B. G. J. Knols, G. Voigt, and J. Hendrichs, "Conceptual framework and rationale," Malaria Journal, vol. 8, supplement 2, p. 1, 2009.

[34] R. H. Dadd, J. E. Kleinjan, and S. M. Asman, "Eicosapentaenoic acid in mosquito tissues: differences between wild and laboratory-reared adults," Environmental Entomology, vol. 17, no. 2, pp. 172-180, 1988.

[35] B. J. Huho, K. R. Ng'habi, G. F. Killeen, G. Nkwengulila, B. G. J. Knols, and H. M. Ferguson, "Nature beats nurture: a case study of the physiological fitness of free-living and laboratoryreared male Anopheles gambiae s.l," Journal of Experimental Biology, vol. 210, no. 16, pp. 2939-2947, 2007.

[36] F. M. Mutuku, J. A. Alaii, M. N. Bayoh et al., "Distribution, description, and local knowledge of larval habitats of Anopheles gambiae s.l. in a village in western Kenya," American Journal of Tropical Medicine and Hygiene, vol. 74, no. 1, pp. 44-53, 2006. 

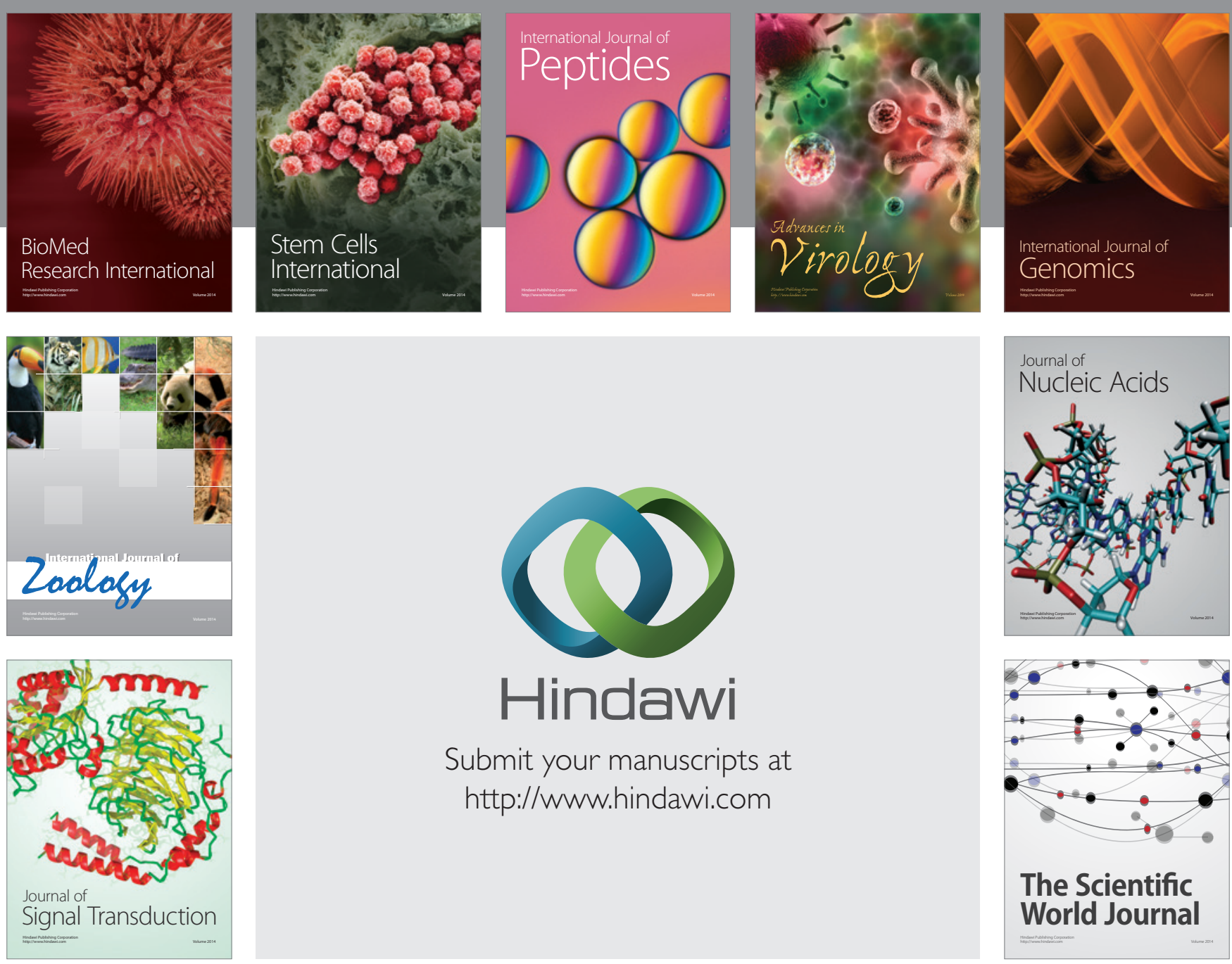

Submit your manuscripts at

http://www.hindawi.com
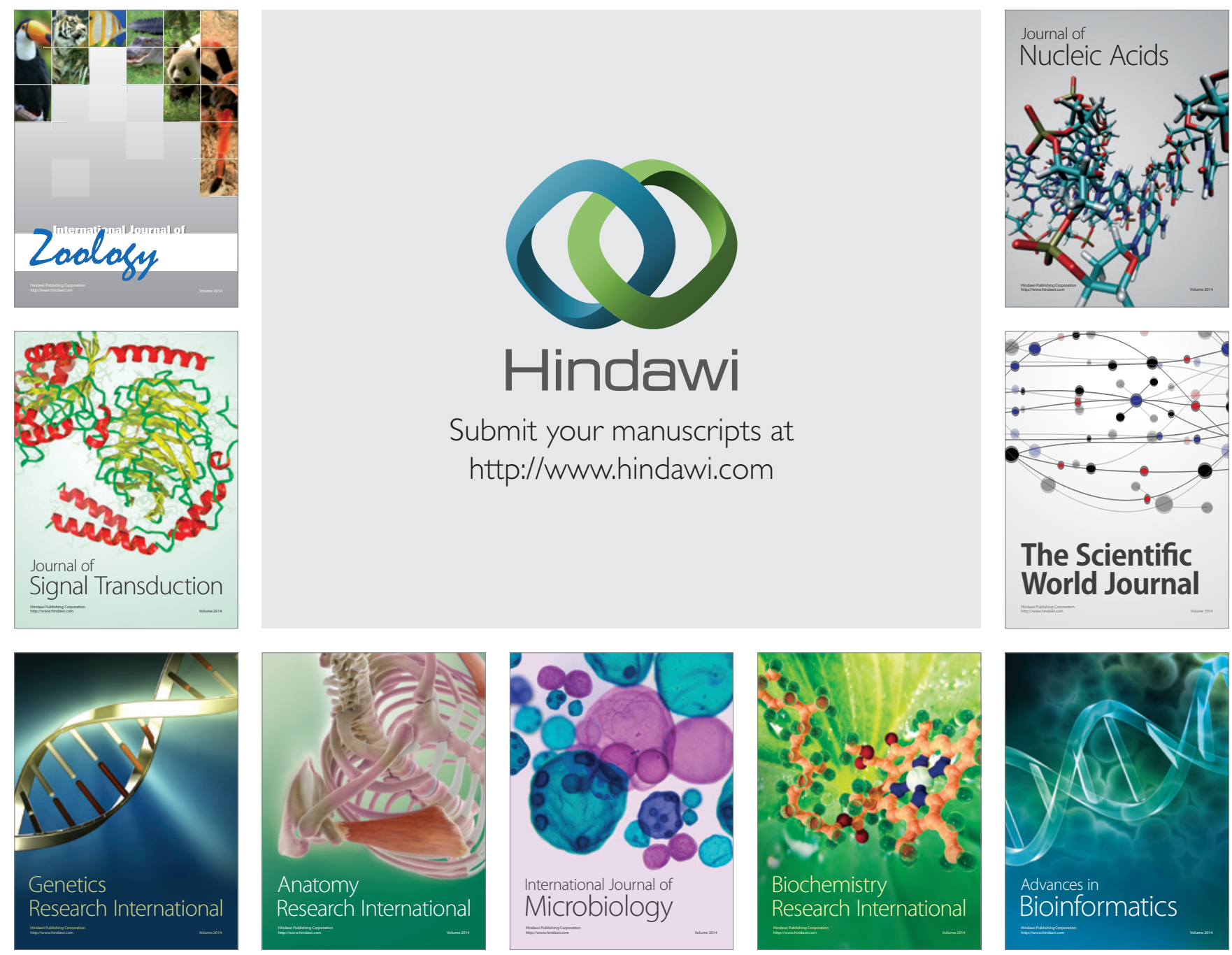

The Scientific World Journal
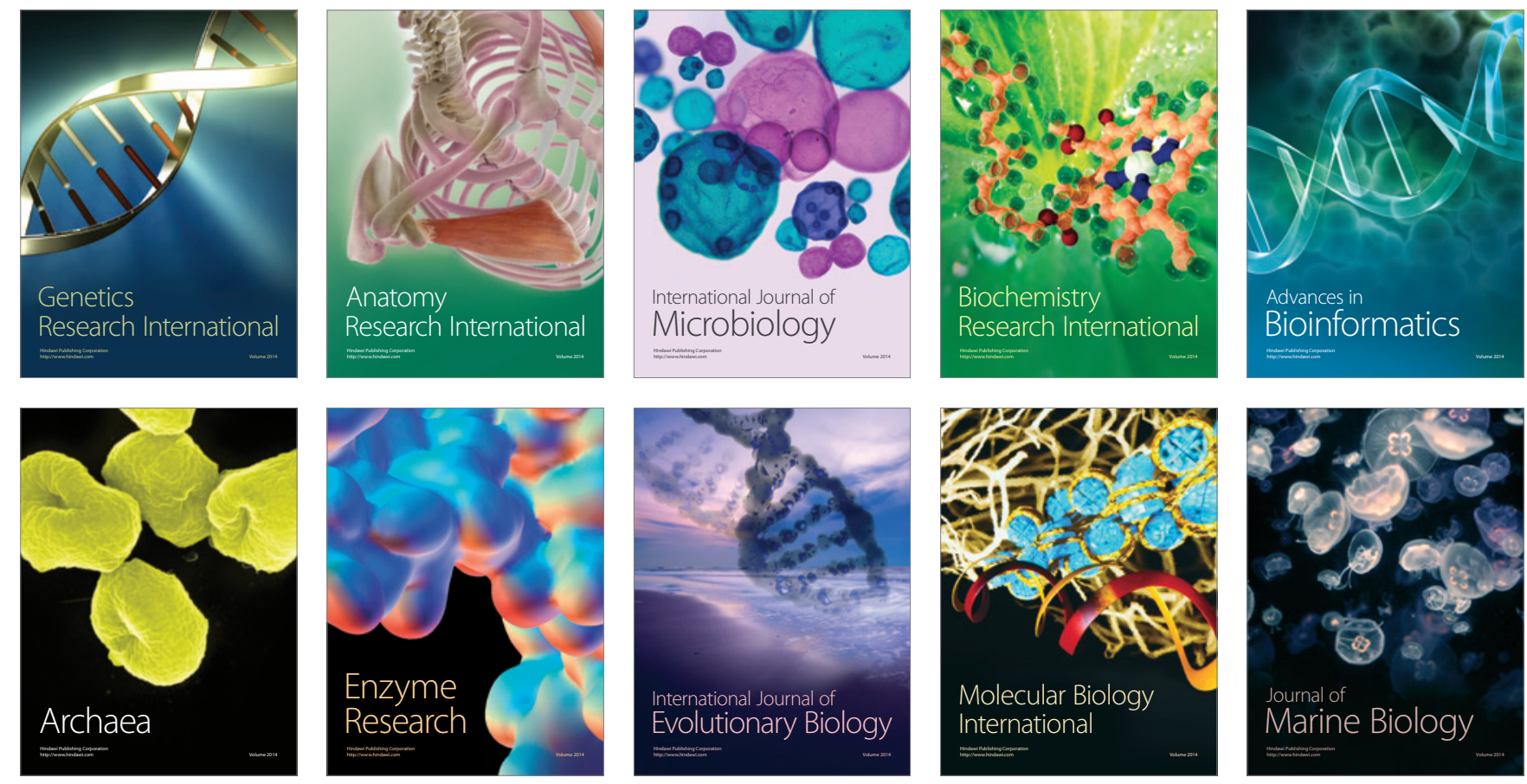